IV. Aus der anatomischen Abtheilung (Prosektor: Prof. Dr. Hansemann) und der inneren Abtheilung (Dirig. Arzt: Prof. Dr. Krönig) des städtischen Krankenhauses am Friedrichshain in Berlin.

\title{
Die experimentelle Lumbalpunktion zum Nachweis von Tuberkelbacillen.
}

Von Dr. H. Hellendall, früherem Assistenzarzt.

Der Nachweis von Tuberkelbacillen in der Lumbalflüssigkeit bei tuberkulöser Meningitis fällt nicht immer positiv aus; das ist eine Erfahrung, welche von allen Autoren, die über diese Frage gearbeitet haben, gemacht worden ist. Durch Verimpfung in die Bauchhöhle des Meerschweinchens hat man den tuberkulösen Charakter der Lumbalflüssigkeit in Fällen, wo das mikroskopische Präparat nichts Positives ergab, festgestellt. Neuerdings hat Langner durch Züchtung von Tuberkelbacillen der Lumbalflüssigkeit, indem er diese selbst als Nährboden benutzte, die Meningitis als eine tuberkulöse charakterisiren könnén. 1)a auch mir nicht selten der Nachweis der Tuberkelbacillen in der Lumbalflüssigkeit durch das mikroskopische Präparat misslang, so kam ich auf den Gedanken, eine tuberkulöse Hirnhautentzündung bei Meerschweinchen durch Lumbalflüssigkeit zu erzeugen, welche an tuberkulöser Meningitis verstorbenen Kindern unmittelbar post mortem entnommen worden war. Der Zweck dieser Versuche war, falls ein positives Ergebniss in wenigen Tagen eintrat, auch Material, welches während versehiedener Stadien dieser Krankheit dem Lebenden entnommen werden sollte, zu benutzen und so durch experimentelle Erzeugung einer tuberkulösen Meningitis bei dem für Tuberkulose so empfindlichen Meerschweinchen in Frühstadien dieser Krankheit am anatomischen Präparat die Diagnose dieser Krankeit zu stellen. Das Resultat meiner Versuche ist nun aber ein ganz anderes geworden, als ich erwartet hatte. Zunächst über die Methode einige Worte.

Ich habe sowohl die Atlanto-occipital- als auch die Lumbalgegend zam Einstich gewählt. Während ich sofortigen Exitus bei der ersteren Methode erlebte, ist mir das an der gewöhnlichen Stelle der Lumbalpunktion nicht vorgekommen. Ich kann also nur dazu rathen, wenn man am Meerschweinchen operirt, diese Stelle zu wählen. Wer diese Versuche macht, thut gut, vorher an der Wirbelsäule eines getödteten Meerschweinchens sich über die Lage der immer etwas von unten nach oben schräg einzuführenden Nadel zu orientiren. Auf diese Weise lernt er dann auch, dass man nicht tiefer als ca. $1 \mathrm{~cm}$ einzustechen braucht, dann ist man bereits im Wirbelkanal. Durch Aspiration gelang es mir nicht, Flüssigkeit aus dem Wirbelkanal des Meerschweinchens zu gewinnen. Eine Aspiration nach dem Einstich garantirt also nicht, dass die Nadel in den Wirbelkanal eingedrungen ist. Diese Sicherheit gewinnt man einmal durch den Vorversuch am Skelett und ferner durch die Beobachtung plötzlich mit der Injektion der Flüssigkeit eintretender Gehirn erscheinungen. Man injizirt mit ganz langsamem Druck $2 \mathrm{ccm}$ der Cerebrospinalflüssigkeit. $3 \mathrm{ccm}$ ist zuviel. Der Druck darf nur ganz gering sein, sodass eine Zeit von mehreren Minuten über der Injektion vergeht. Die Nadel der Spritze muss recht fein sein. Eine Pravaz'sche Spritze reicht aus. Das auf dem Brett fixirte Meerschweinchen wird zweckmässiger Weise bei dem Einstich in die linke Hand genommen und der Rücken, namentlich der Lumbaltheil, etwas stärker dorsal convex gekrümmt, es gelingt dann leicht, auf der abrasirten und desinfizirten Lumbalgegend die Processus spinosi abzutasten und zwischen 
viertem und fünftem Wirbel die Nadel der Spritze einzustechen. Dann erst wird die Spritze aufgesetzt und $2 \mathrm{ccm}$ Flüssigkeit injizirt. Die schweren Erscheinungen, die auftraten, wurden erst beobachtet nach der Injektion der Flüssigkeit. Dennoch untersuchte ich durch Eröffnung des Wirbelkanals, ob durch den Stich Theile des Rückenmarks getroffen worden seien, und ich konnte mich niclit überzeugen, dass dies der Fall war. Um eine Läsion sicher auszuschliessen, versuchte ich am todten Meerschweinchen von dem Caudaltheil der Wirbelsäule, dicht an der Hinterfläche derselben bleibend, in den Wirbelkanal einzudringen. Ich bin davon abgekommen, da in den meisten Fällen die Nadel, wenn sie eindringt, nicht in den Wirbelkanal, sondern in der Muskulatur zu liegen kommt, sodass also dieses Vorgehen zu viele Unsicherheiten bietet.

Die Erscheinungen, die unmittelbar nach demEinströmen der Flüssigkeit in den Wirbelkanal eintraten, waren folgende: Während das Thier im Momente des Einstichs lebhaft zappelte und um sich biss, beobachtete man während und nach dem Einströmen der Flüssigkeit ein deutliches Nachlassen des Widerstandes, die Augen traten vor und verdrehten sich, das namentlich nach dem Einstich erfolgte Schreien hörte auf, die hinteren Extremitäten sanken schlaff herab; manchmal waren beide oder die eine vordere Extremität grelähmt, während die Atllmung und die Herzthätigkeit fortdanerten. Das Thier lag wie leblos da. Nicht inımer war das Bild der Lähmung so deutlich, oft war es mit dem Bilde der Betäubung gethan, welche doch eine ganze Weile, drej- bis vier Minuten, vorhielt. Erst allmählich kam das Thier wieder zu sich und machte mühsame Versuche sich fortzuschleppen. Die beiden Extremitäten blieben manchmal mehrere Tage gelähmt, meist aber war die Lüllmung und die Betäubung nach 24 Stunden vorüber. Die Nahrungsaufnalıme war dann ganz in Ordnung. Immer aber bildeten sich die paralytischen Erscheinungen völlig zurück, sodass das Thie von einem gesınden oberflächlich kaum zu unterscheiden war. Ich hatte den Eindruck, dass die Betänbung bei ganz langsamer Injektion zurücktrat, während die Paraplegie fast immer mehr oder weniger dentlich vorhanden war.

Tabelle über die Experimente.

\begin{tabular}{|c|c|c|}
\hline Tag der Impfung & $\begin{array}{l}\text { Menge des } \\
\text { Materials }\end{array}$ & Reaktion und Verlauf \\
\hline 1. 15. December 1899 & $2 \mathrm{ccm}$ & Keine Lähmung. Geringe Betäubung. \\
\hline 2. 4. Januar 1900 . & $3 \mathrm{ccm}$ & $\begin{array}{l}\text { Rechtseitige Zuckungen, dann Läh- } \\
\text { mung, mehrere Tage andauernd, } \\
\text { dann sich zurückbildend und Wohl- } \\
\text { befinden. }\end{array}$ \\
\hline 3. 6. Januar 1900 & $1 \mathrm{ccm}$ & $\begin{array}{l}\text { Injektion an der Halswirbelsäule. Be- } \\
\text { täubung, keine Lähmung. }\end{array}$ \\
\hline 4. 28. Januar 1900. & $2 \mathrm{ccm}$ & Geringe Betäubung. \\
\hline 5. 2. Februar 1900 . & $2 \mathrm{ccm}$ & wie 4. \\
\hline 6. 8. März 1900 & $2 \mathrm{ccm}$ & $\begin{array}{l}\text { Starke Betäubung. Fast völlig leblos. } \\
\text { Allmähliche Erholıng. Dann Para- } \\
\text { plegia totalis. Nach } 2 \times 24 \text { Stun- } \\
\text { den vorbei. }\end{array}$ \\
\hline 7. 8. März 1900 & $\left|\begin{array}{c}\text { von dem- } \\
\text { selben } 2 \mathrm{ccm}\end{array}\right|$ & $\begin{array}{l}\text { Paraplegie der Beine. Keine Betäu- } \\
\text { bung. }\end{array}$ \\
\hline
\end{tabular}

Ueber den Verlauf der Krankheit, die nun eintrat, möchte ich hervorheben, dass schwerere cerebrale Störungen nicht beobachtet wurden, insbesondere meningitische Symptome in sämmtlichen Fällen nicht constatirt werden konnten. Der Tod trat in den sieben Fällen, welche sämmtlich mit Lumbalflüssigkeit von anatomisch nachgewiesener tuberkulöser Meningitis geimpft waren, ausnahmslos ein. 5 Meerschweinchen starben an allgemeiner Miliartuberkulose, 1:3 Wochen, 1:6 Wochen, $3: 8$ Wochen nach dem Tage der Impfung. Zwei Thiere waren nicht ganz 24 Stunden nach dem Eingriffe todt. Diese boten anatomisch nichts weiteres, als Hyperämie des Gehirns und Rückenmarks. Bei dem Meerschweinchen, welches drei Wochen nach der Impfung starb, waren die geringsten anatomischen Veränderungen der allgemeinen Tuberkulose vorhanden. Hier war die in der Nähe des Einstiches gelegene retroperitoneale Lymphdrüse stark geschwollen und verkäst. Es waren zahlreiche Tuberkel in der Milz, die aber sonst überall fehlten, und die eigentliche Todesursache war eine schwere Bronchopneumonie. Im Gehirn und Rückenmark war nichts Besonderes. In dem Falle, wo der Tod nach sechs Wochen eintrat, fanden sich massenhaft Tuberkel in der Milz und Leber. Die Miliartuberkulose war am ausgebreitetsten in den drei Fällen, wo der Exitus nach acht Wochen erfolgte. In zweien konnte man verfolgen, wie die Lymphdrüsen, die auf der Wirbelsäıle liegen, perlschnurartig sich aneinander reihten und verkäst waren und sich von der Nähe der Injektionsstelle in die Milzgegend fortsetzten. Auch die Leber war befallen. In den Lungen Bronchopneumonie.

In dem dritten Falle, der der interessanteste ist, fand sich eine vollkommene Durchsetzung mit kleinen und grossen verkästen Tuberkeln der retroperitonealen und peribronchialen Lymphdrüsen, der Milz, Leber und namentlich beider Lungen in ihrer ganzen Ausdehnung. Ausserdem konnte ich an der Injektionsstelle im Wirbelkanal käsigen Eiter constatiren, in welchem sich mikroskopisch sehr viele Tuberkelbacillen und mono- und polynukleäre Elemente fanden. Das Gehirn und Rückenmark, waren unbetheiligt.

In den Fällen 1, 2, 4 (siehe Tabelle) ergab die mikroskopische Untersuchung der Organe, speziell des Gehirns, die Verbreitung des tuberkulösen Prozesses in allen anderen Or ganen, während das Gehirn und die Oberfläche desselben vollständig frei blieben.

Hinsichtlich der Ausbreitung des tuberkulösen Prozesses bei

\section{Tod \\ Anatomischer Befund}

4. Februar
1900 Miliartuberknlose: Leber, Milz. Hyperämie des Gehirns.

1. März Miliartuberkulose: Leber, Milz, Lungen. Retroperito1900 neale und peribronchiale Lymphdriisen verkäst. Käsiger Eiter im Wirbelkanal an der Injektionsstelle. Darin reichlich Tuberkelbacillen. Gehirn und RiickenDarin reichlich Tuberkelbacill
mark ohne Besonderheiten.

Nach 24 Stunden

31. März Miliartuberkulose: Milz; Leber; Lymphdriisen auf der 1900 Wirbelsäule perlschnurartig, verkäst. Bronchopneumonie. Gehirn und Rückenmark ohne Besonderheiten.

wie $4 . \quad$ wie 4.

27. März Grosse käsig eitrige Lymphdrüsen retroperitoneal. Zahl1900 reiche Tuberkeln in der Milz. Bronchopneumonia gravis. Gehirn und Riickenmark ohne Besonderheiten.

9. März 1900 Hyperämie des Gehirns und Rückenmarks.

dieser experimentellen Methode ist zu schliessen, dass die Tuberkelbacillen, welche in den Lymphsack des Wirbelkanals injizirt wurden, auf Lymphbahnen in die retroperitonealen Lymphdrüsen abfiltrirt werden, hier Boden fassen, um dann in die Milz und Leber transportirt zu werden, und so in die Lungen gelangen. Dieser Verbreitungsmodus ist allen experimentellen Resultaten gemeinsam, wie Cornet (Tuberkulose) S. 64, 65 hervorhebt, und reihen sich diese Versuche deshalb den bisherigen in dieser Beziehung harmonisch an.

Das Resultat dieser Untersuchungen ist nicht dies, dass es mir gelungen ist, am Meerschweinchen mit dem genannten Material eine tuberkulöse Meningitis zu erzeugen. Der Gedanke, eine am Meerschweinchen experimentell erzeugte tuberkulöse Meningitis zur Diagnose der Frühstadien dieser Krankheit am Lebenden zu benutzen, konnte daher leider von mir nicht verwirklicht werden. Es ist vielmehr eine Miliartuberkulose erzeugt worden. - Mit dieser Frage hat sich bereits Martin (Soc. de biologie 1898, Séance 5. März, pag. 273 und 19. November, pag. 1068) beschäftigt.

Er hat 1. nicht die eigentliche Lumbalpunktion gemacht, sondern den Atlanto-occipitalweg eingeschlagen, diesen aber später $\mathrm{zu}$ Gunsten der Trepanation aufgegeben.

2. Es ist ihm gelungen, mit Reinkulturen von Tuberkelbacillen eine anatomisch charakterisirte tuberkulöse Meningitis bei Meerschweinchen und Kaninchen zu erzeugen.

3. Er hat auch mit tuberkulöser Lumbalflüssigkeit auf diesem Wege Meerschweinchen getötet, bringt aber keine Angabe über einen anatomischen Nachweis einer tuberkulösen Meningitis.

Auch M. A. Sicard und Péron haben Experimente in dieser Richtung gemacht,

M. A. Sicard in der Sitzung vom 29. October 1898 in der Société de biologie (Semaine médicale 1898, pag. 447): Communique un certain nombre d'expériences faites sur le chien, qui tendent à prouver, que la cavité sous-arachnoidienne est très tolérante à l'égard des corps non toxiques, que les subsistances abandonnées à son intérieur peuvent 
diffuser et être resorbées, qu'elles agissent plus efficacement, que par la voie veineuse ou sous-cutanée, et que au point de vue thérapeutique l'injection sous-arachnoidienne lombaire doit être préferée à l'injection sous-arachnoidienne crânienne. L'orateur a essayé d'entraver par ce moyen l'éclosion d'une tuberculose: à cet effet il a soumis il y a six mois un lot de quatre chiens après inoculation sous-arachnoidienne de cultures de Koch à l'injection sous-arachnoidienne par la voie lombaire de 5 à $10 \mathrm{ccm}$ d'huile jodoformée.

Cette injection préventive a été renouvelée tous les quinze jours environ. Les animaux temoins ont succombé, les chiens ayant reçu de l'huile jodoformée ont jusqu'à présent résisté."

Péron (Archives générales de médecine 1898, II, pag. 570) berichtet über die experimentelle tuberkulöse Meningitis bei Hunden: „L'autopsie soigneusement faite démontre qu'il n'y a pas seule manifestation tuberculeuse en dehors de l'encéphale, que tout s'est passé à la surface des méninges. J'ai cherché envain au microscope un tubercule dans les organes de l'animal."

Sicard hat also die experimentelle Lumbalpunktion beim Hunde benutzt. $\mathrm{Ob}$ er eine anatomisch nachgewiesene tuberkulöse Meningitis erzeugt hat, geht aus obigem nicht hervor. Er hat Reinkulturen von Tuberkelbacillen angewandt. Péron hat, wie er angiebt, das Verfahren von Magendie angewandt und am Hunde eine anatomisch nachgewiesene tuberkulöse Meningitis mit Reinkulturen von Tuberkelbacillen hervorgerufen.

$\mathrm{Nach}$ Martin, Sicard und Péron kann man also beim Meerschweinchen, Kaninchen und Hunde eine anatomisch sichere tuberkulöse Meningitis mit Reinkulturen von Tuberkelbacillen durch verschiedene Methoden erzeugen. Ob man dies Ziel auch durch Injektion tuberkulöser Lumbalflüssigkeit erreicht, scheint mir aus obigem nicht hervorzugehen.

Mir ist es nicht gelungen, vermittels der typischen Lumbalpunktion durch Injektion tuberkulöser Lumbal flüssigkeit am Meerschweinchen eine tuberkulöse Meningitis hervorzurufen. Es trat vielmehr eine allgemeine Miliartuberkulose ein.

Ich habe mir den Einwand zu machen, dass ich mit der Nadel vielleicht iiberhaupt nicht im Intrameningealraum gewesen bin und dass deshalb eine Meningitis nicht hat erzeugt werden können. Da es mir nie gelang, Lumbalflüssigkeit zu aspiriren, nachdem die Nadel eingestochen, so könnte es scheinen, als ob der Einwand berechtigt wäre. Ich gebe auch zu, dass ich denselben völlig zu entkräften nicht in der Lage bin. Ich möchte aber darauf hinweisen, dass die Lähmungserscheinungen, die sich allemal zurückbildeten, verbunden mit schwersten Gehirnerscheinungen, die im Momente des Einströmens der Flüssigkeit, nicht aber im Augenblick der Einführung der Nadel eintraten, eine plötzliche Gehirndrucksteigerung als Erklärung nahelegen. Ich darf ferner betonen, dass in einem Falle, wo eine allgemeine Miliartuberkulose eintrat, wo aber das Gehirn nach makro- und mikroskopischer Betrachtung sich als intakt herausstellte, an der Stelle der Injektion im Wirbelkanal käsiger Eiter sich fand, in dem massenhaft Tuberkelbacillen nachgewiesen werden konnten.

Jedenfalls schienen mir meine Resultate wohl der Mittheilung werth, da sie geeignet sind, die Bedeutung des Nachweises der Tuberkelbacillen durch experimentelle Lumbalpunktion erkennen zu lassen.

Der mikroskopische Nachweis der Tuberkelbacillen in der Lumbalflüssigkeit gelingt, wie bereits oben bemerkt, nicht immer. Das negative Resultat, welches von fast allen Beobachtern (Litteratur: S. Slawyk und Manicatide [Berliner klinische Wochenschrift 1890 , S. 392], Zur bacillären Diagnose der Meningitis tuberculosa; Schwarz [Deutsches Archiv für klinische Medicin Bd. LX], Zur klinischen Würdigung der Diagnose der tuberkulösen Meningitis vermittels der Lumbalpunktion) in einer Reihe von Fällen zugegeben wird, ist nun aber weder für noch gegen die Diagnose „tuberkulöse Meningitis“ zu verwerthen. Aber es legt den Gedanken nahe, andere Methoden zu suchen, die den Tuberkelbacillus nachweisen.

Das Thierexperiment, und zwar die intraperitoneale Einverleibung der Lumbalflüssigkeit beim Meerschweinchen, ist in solchen Fällen mit Erfolg angewendet worden. Bernheimer und Moser (Wiener klinische Wochenschrift 1897, S. 473) haben bei 60 Fällen von tuberkulöser Meningitis $14 \mathrm{mal}$ den Tuberkelbacillus nicht nachweisen können. In diesen 14 Fällen haben sie noch $12 \mathrm{mal}$ durch intraperitoneale Einverleibung der Fliissigkeit am Meerschweinchen den tuberkulösen Charakter derselben feststellen können. Zweimal blieb das Thier gesund. In 8 Fällen dauerte es 5 Wochen bis 4 . Monate bis zum Tode ( 2 mal 5 Wochen, 2 mal 7 Wochen, 2 mal 8 Wochen, 1 mal 3 Monate, 1 mal 4 Monate) In 4 Fällen wurde das Thier getödtet ( 1 mal nach 6,2 mal nach 8 Wochen, 1 mal nach 6 Monaten). $1-4 \mathrm{ccm}$ wurden injizirt.

Marfan (Sammelwerk: Grancher, Traité des maladies de l'enfance tome IV, pag. 396) hatte mit der intraperitonealen Methode trotz positiver Obduktionsbefunde negative Thierversuchsresultate.

Slawyk und Manicatide (l. c.) führen Marfan's negativen Resultate darauf zurück, dass zu wenig Material injizirt sei. Man muss 4 ccm injiziren.

Sie haben 16 mal den Tuberkelbacillus in der Lumbalflüssigkeit mikroskopisch nachgewiesen. In drei negativen Fällen hatten sie mit der intraperitonealen Methode positiven Thierversuch. Sie haben die Meerschweinchen nach 5 Wochen getödtet.

Negative Resultate mit dieser Methode lassen zwei Erklärungen zu: Entweder war die verimpfte Flüssigkeit, wie das bei dem geringen Gehalt des ganzen Materials an Tuberkelbacillen sehr möglich ist, frei von Bakterien, oder die spärlichen Tuberkelbacillen sind, wie Langner glaubt, vom Meerschweinchen iiberwunden worden (Prager Zeitschrift für Heilkunde 1899, S. 352).

Jedenfalls geht aus diesen Versuchen hervor, dass zum Nachweis der Tuberkelbacillen in der Lumbalflussigkeit der Thierversuch eine exaktere Methode darstellt als das mikroskopische Präparat.

Gegenüber der in traperito nealen Einverleibung der Lumbalflüssigkeit ist hervorzuheben, dass bei der Einverleibung derselben durch experimentelle Lumbalpunktion eine raschereWirkung einzutreten scheint, wenigstens erfolgte in den sieben erwähnten Fällen der Tod spätestens in acht Wochen. Als ein weiterer Vortheil erscheint die geringere Menge, welche hinreicht, einen positiven Thierversuch zu erreichen. Bei der experimentellen Lumbalpunktion genügen $2 \mathrm{ccm}$. Bei der intraperitonealen Methode scheinen $4 \mathrm{ccm}$ (siehe oben) nothwendig zu sein. Im Leben kann man aber nicht immer 4 ccm auf einmal entnehmen.

Zur Erklärung einer rascheren Wirkung auf dem Wege der Lumbalpunktion mag betont werden, dass Langner (l. c.) die Lumbalflüssigkeit als vorzüglichen Nährboden für Tuberkelbacillen (im Brutofen) erkannt und nachgewiesen hat.

Hier wird aber die Lumbalflüssigkeit innerhalb des leb enden Thieres als Nährboden benutzt, und zwar des Meerschwein chens, welches für den Tuberkelbacillus so überaus empfindlich ist.

Dadurch sind drei Faktoren gegeben, welche es erklären können, dass bei der experimentellen Lumbalpunktion eine raschere Wirkung einzutreten scheint, als das von der intraperitonealen Methode berichtet wird.

Ich möchte also glauben, dass die experimentelle Lumbalpunktion zum Nachweis derspärlichen Tuberkelbacillen in der Lumbalflüssigkeit vor der intraperitonealen Methode Vorzüge hat, mindestens aber ihr gleichwerthig ist.

Langner (l. c.) züchtet den Tuberkelbacillus in der Lumbalflüssigkeit, wie bereits bemerkt. Er will eine rasche Vermehrung derselben sogar in acht Tagen schon beobachtet haben. Er hat dadurch eine Methode gefunden, die sicherlich die grösste Beachtung verdient. In den fünf Fällen jedoch unter im ganzen 21, in denen das mikroskopische Präparat frei von Tuberkelbacillen war, war das kulturelle Ergebniss (der Tabelle nach zu urtheilen!) für die klinische Verwerthung zu spät positiv: 1. einen Tag vor dem Tode; 2. 11 Tage nach dem Tode; 3. zwei Wochen nach dem Tode; 4. vier Wochen nach dem Tode; 5. fünf Wochen nach dem Tode.

Bis jetzt hat also die Methode Langner's, die billiger und schneller ist, als das Thierexperiment, keine wesentlichen Vorzüge vor diesem hinsichtlich der Diagnosenstellung am Krankenbet gehabt.

Eine allgemeine Bedeutung kann die experimentelle Lumbalpunktion vielleicht gewinuen, wenn man die Methode überhaupt benutzen will zum Nachweis von spärlichen Tuberkelbacillen in irgend einer sonst sterilen Flüsigkeit. Sie verdient also zum Beispiel Berücksichtigung, wenn man in verdächtigen Pleura- und Bauchhöhlenexsudaten den Tuberkelbacillus constatiren möchte.

Bruns und Levy, Deutsche medizinische Wochenschrift 
1900, No. 9, S. 141, haben geringe Mengen Tuberkelbacillen im Sputum, nachdem dasselbe durch bestimmte Methoden coccenfrei gemacht war, aufgeschwemmt, dem Meerschweinchen intraperitoneal einverleibt und das Thier tuberkulös gemacht. Auch in dieser Richtung liegt vielleicht eine klinische Bedeutung der experimentellen Lumbalpunktion. 